University of Nebraska - Lincoln

DigitalCommons@University of Nebraska - Lincoln

Nebraska Cooperative Fish \& Wildlife Research Nebraska Cooperative Fish \& Wildlife Research Unit -- Staff Publications

2012

\title{
Predictions and retrodictions of the hierarchical representation of habitat in heterogeneous environments
}

Jurek Kolasa

McMaster University, kolasa@mcmaster.ca

Craig R. Allen

USGS Nebraska Cooperative Fish and Wildlife Research Unit, University of Nebraska, callen3@unl.edu

Jan Sendzimir

International Institute for Applied Systems Analysis, Laxenburg, Austria, sendzim@iiasa.ac.at

Craig A. Stow

NOAA - Great Lakes Environmental Research Laboratory, Ann Arbor, MI, craig.stow@noaa.gov

Follow this and additional works at: https://digitalcommons.unl.edu/ncfwrustaff

Part of the Other Environmental Sciences Commons

Kolasa, Jurek; Allen, Craig R.; Sendzimir, Jan; and Stow, Craig A., "Predictions and retrodictions of the hierarchical representation of habitat in heterogeneous environments" (2012). Nebraska Cooperative Fish \& Wildlife Research Unit -- Staff Publications. 101.

https://digitalcommons.unl.edu/ncfwrustaff/101

This Article is brought to you for free and open access by the Nebraska Cooperative Fish \& Wildlife Research Unit at DigitalCommons@University of Nebraska - Lincoln. It has been accepted for inclusion in Nebraska Cooperative Fish \& Wildlife Research Unit -- Staff Publications by an authorized administrator of DigitalCommons@University of Nebraska - Lincoln. 


\title{
Predictions and retrodictions of the hierarchical representation of habitat in heterogeneous environments
}

\author{
Jurek Kolasa $^{\mathrm{a}, *}$, Craig R. Allen ${ }^{\mathrm{b}}$, Jan Sendzimir ${ }^{\mathrm{c}}$, Craig A. Stow ${ }^{\mathrm{d}}$ \\ a Department of Biology, McMaster University, Hamilton, Ontario, Canada \\ ${ }^{\mathrm{b}}$ U.S. Geological Survey - Nebraska Cooperative Fish E Wildlife Research Unit, School of Natural Resources, 423 Hardin Hall, University of Nebraska, Lincoln, NE 68583, USA \\ ' International Institute for Applied Systems Analysis, Laxenburg, Austria \\ d NOAA - Great Lakes Environmental Research Laboratory, Ann Arbor, MI, USA
}

\section{A R T I C L E I N F O}

\section{Article history:}

Available online $\mathrm{xxx}$

\section{Keywords:}

Hierarchical habitat structure

Predictions

Species abundance

Discontinuities in ecological traits

Allometry

Comparative population variability

\begin{abstract}
A B S T R A C T
Interaction between habitat and species is central in ecology. Habitat structure may be conceived as being hierarchical, where larger, more diverse, portions or categories contain smaller, more homogeneous portions. When this conceptualization is combined with the observation that species have different abilities to relate to portions of the habitat that differ in their characteristics, a number of known patterns can be derived and new patterns hypothesized. We propose a quantitative form of this habitat-species relationship by considering species abundance to be a function of habitat specialization, habitat fragmentation, amount of habitat, and adult body mass. The model reproduces and explains patterns such as variation in rank-abundance curves, greater variation and extinction probabilities of habitat specialists, discontinuities in traits (abundance, ecological range, pattern of variation, body size) among species sharing a community or area, and triangular distribution of body sizes, among others. The model has affinities to Holling's textural discontinuity hypothesis and metacommunity theory but differs from both by offering a more general perspective. In support of the model, we illustrate its general potential to capture and explain several empirical observations that historically have been treated independently.
\end{abstract}

(C) 2012 Published by Elsevier B.V.

\section{Introduction}

Recognition that ecological phenomena occur at various scales has informed ecological research for over 20 years. Allen and Starr (1982) made the first comprehensive review of the conceptual and practical issues associated with the multiscale nature of ecology, which they centered on a hierarchical view of ecological systems. Following from this initial work, the scale-dependence of ecological patterns became a major focus in ecology, as well as the interaction of processes at various scales (e.g., Davies et al., 2005; Sandin and Pacala, 2005). The application of this view to research problems of community and ecosystem ecology was particularly successful with respect to interpretation of ecological patterns but less so in formulating testable hypotheses. Instances where such hypotheses have been formulated are limited; Kolasa (1989) postulated the existence of discontinuities in community structure and Holling (1992) proposed a link between landscape texture and body size distributions (textural discontinuity hypothesis). This modest record contributes to a more limited recognition and

\footnotetext{
* Corresponding author at: Department of Biology, McMaster University, 1280 Main Str W, Hamilton, Ontario L8S 4K1, Canada.

E-mail address: kolasa@mcmaster.ca (J. Kolasa).
}

understanding of the hypotheses and predictions arising from the multiscale perspective than they deserve to have. However, the hierarchical view of ecological systems has significant potential for explaining old and predicting new processes, patterns, and relationships. An approach that has the ability to account for observations that had previously required separate and unconnected models - a case of retrodiction - would be particularly useful.

We attempt to address this need by reviewing the predictions of hierarchy theory as applicable to broadly defined community ecology and by generating further testable hypotheses derived from a single conceptual construct. Specifically, we advance a model linking the structure of the environment to structure and dynamics of communities and to properties of their constituent species. First, we describe the model to accommodate various ecological premises. Then we formalize its quantitative potential. Finally, we apply it to a selection of contemporary problems in ecology.

\section{Verbal and graphical model of habitat-species relationship}

\subsection{Habitat structure}

Any habitat, aquatic or terrestrial, is a nested mosaic of smaller habitats such that larger fragments contain smaller subcategories, 


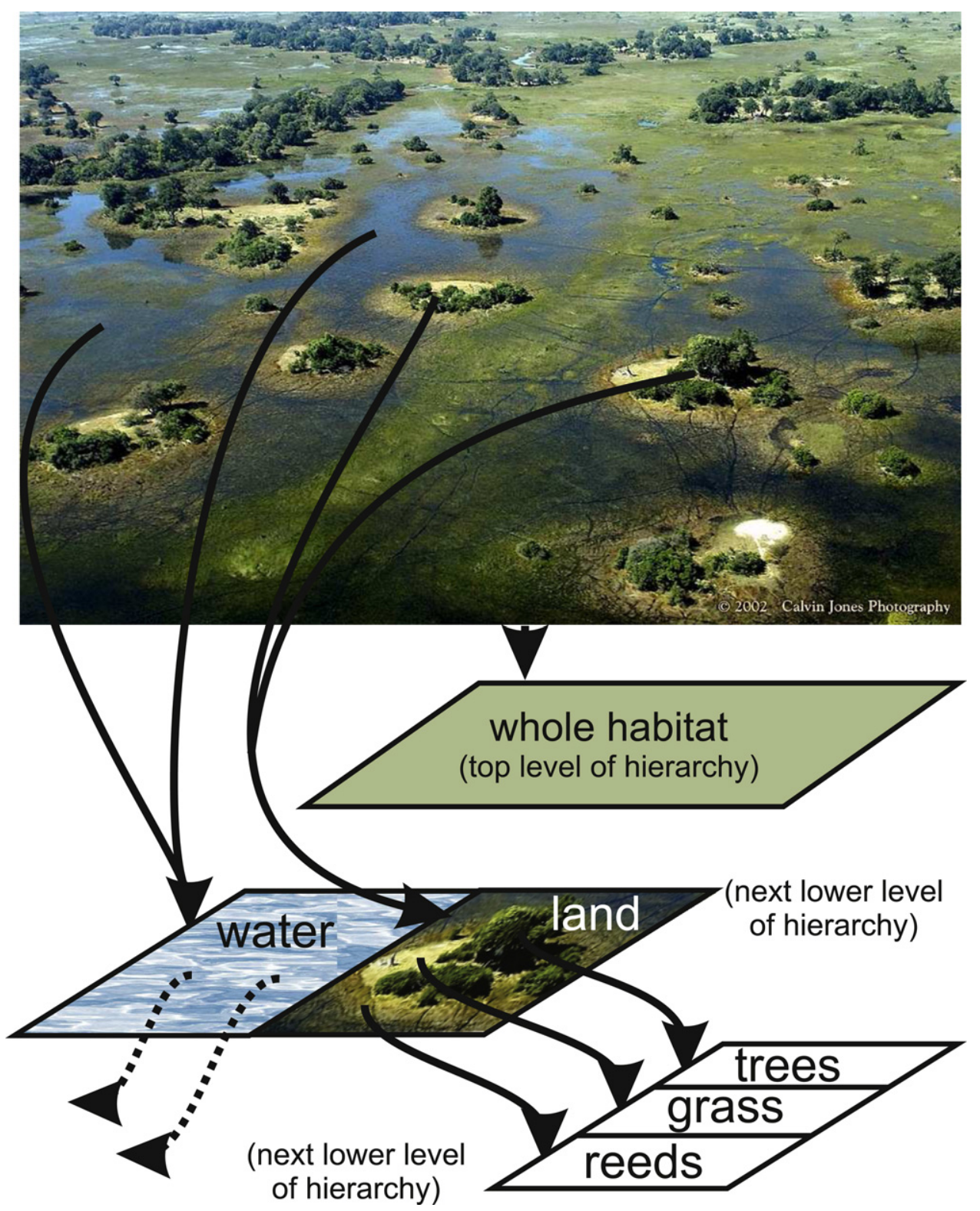

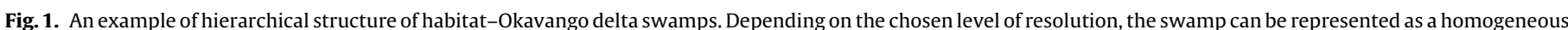

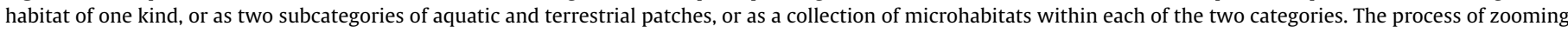
in can continue as desired.

Photograph by Calvin Jones, with permission.

and those in turn are composed of even finer microhabitat subcategories that are even smaller (Fig. 1). Indeed, the metaphor provided by the pictorial example can be extended to other dimensions. Specifically, we assume that habitat is a nested hierarchy of multidimensional volumes (for detailed discussion see Kolasa and Waltho, 1998). Because any habitat dimension except time can be mapped onto space, we interpret the habitat as (1) a hierarchical mosaic of patches with (2) lower-level patches nested within higher-level patches, and (3) with each level having a distinct set of attributes arising from distinct processes (Fig. 1). It is the interaction of such habitat hierarchy with a subset of species from the regional species pool that determines which of these attributes are meaningful and thus worthy of consideration. Also, while this structure is best conceived as multidimensional, it is likely that one or very few dimensions exert major influence on the performance of any single species (Polechová and Storch, 2008). The commonly accepted notion of a limiting factor provides solid logical support for this assumption. If one factor limits abundance and distribution of a population, whether it is a resource or constraint of some other nature, the other factors, by definition, do not play such a role at the same point in time or play roles too minor to consider.

To examine the model properties we use two dimensions only. Two dimensions provide the model with a spatial attribute, although this considerable simplification does not convey any specific configuration of actual habitats in space. The model identifies the total amount of space a particular habitat occupies on average relative to a higher-level habitat unit. Each unit can take various configurations in space. It can occur as a contiguous patch, or it can differ in size and be split to differing degrees (Fig. 2a-c). Regardless of spatial configuration, two habitat types are represented in the model as two subunits (Fig. 2d) that are members of a single, higher level unit. Extending this approach permits representation of the whole habitat as a nested structure of units emerging at increasing levels of resolution (Fig. 3). 


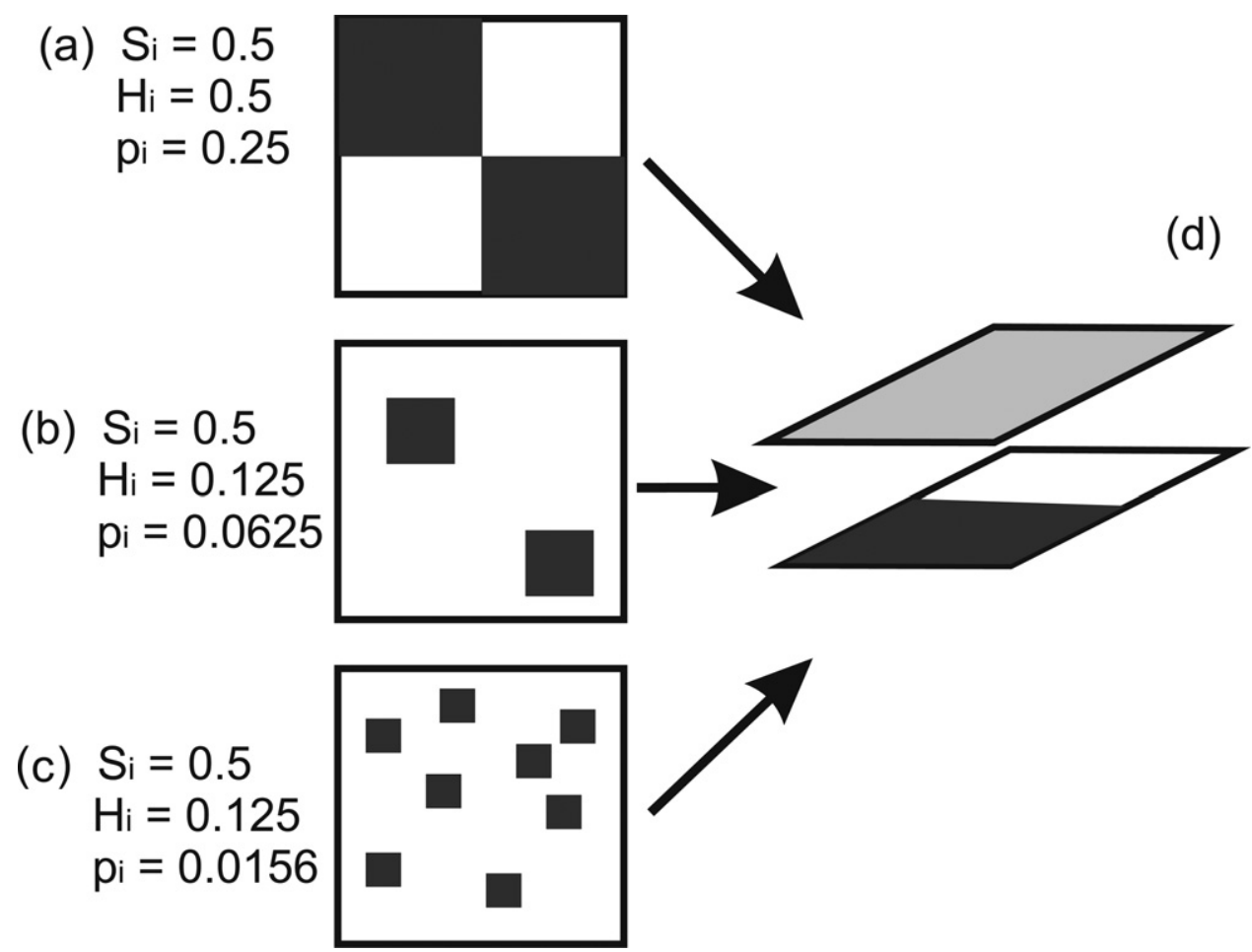

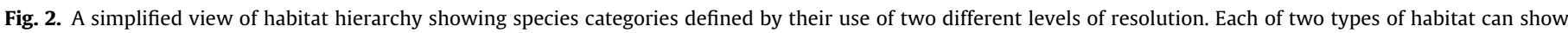

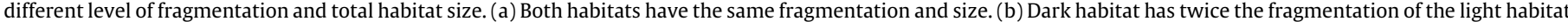

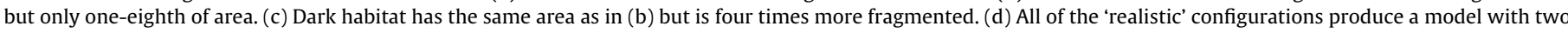

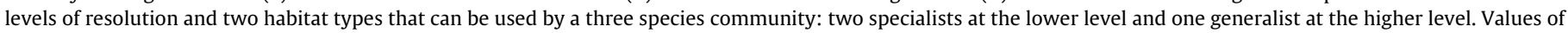
$S_{i}, H_{i}$, and $p_{i}$ are given for the dark type only.

\subsection{Relationship between species and habitat structure}

The relationship between species and habitat assumes the following:

(1) Habitat generalists use the largest habitat units; thus increasing habitat specialization results in the use of progressively smaller habitat units nested within the larger ones and (2) the density of a species varies positively with the habitat unit size and negatively with the degree of unit isolation from other similar units (cf., Kolasa, 1989 for evidence).

Thus, according to the model, the density of generalists will be proportional to the continuous area of the top-level habitat defined by the two dimensions in Fig. 3. The situation is different for specialists at the two lower levels. Although their densities

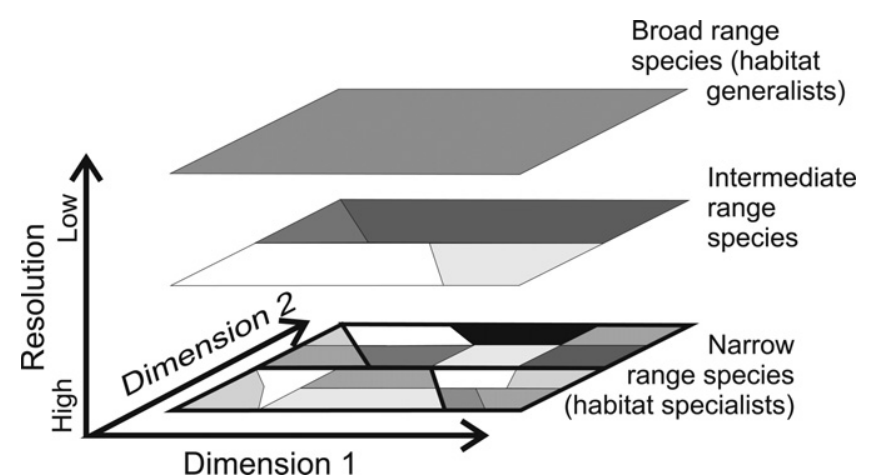

Fig. 3. A hypothetical habitat structure able to accommodate 21 species corresponding to 21 habitat types (from top to bottom: 1,4,16). Note that habitat grain, size, and diversity change with resolution. From species' perspective, the habitat becomes smaller and more fragmented at higher levels of resolution (i.e., lower levels of structure). are related to the area of the habitat used, the expected density is different because their habitats appear as single patches nested within the generalist's habitat. In a habitat mosaic, these habitats are repeated in space but remain separated remain separated from each other by patches that the specialist cannot exploit. The more specialized the species, the greater the average geometrical and ecological distance (e.g., barriers of unsuitable habitats) between suitable units of the environment. Fragmentation of a habitat unit itself (cf., Figs. 1 and 2c) can exacerbate this barrier effect. In a sense, resources available to a specialist become diluted in the patchwork of other habitat units. It is reasonable to assume that a specialist species experiences higher energy and population costs (such as loss of individuals travelling over unsuitable habitat), when it uses patchily distributed habitats. Good dispersers can reduce these costs, but they cannot avoid them entirely. By contrast, in the model, generalists experience a continuum of suitable habitat. There may be several components to the costs experienced by specialists, including energetic costs of travel, mortality during dispersal, decreased foraging efficiency through failure of finding patchily distributed resources, or due to finding and settling in lowquality (sink) patches. From here, we assume these costs reduce a species' density in response to the degree of the habitat fragmentation and amount as experienced at the specialist level as compared to the generalist level. The main benefit accrued from being a habitat generalist comes from the lack of fragmentation costs. Habitat specialization can bring some benefits to a species when the specialization coincides with higher resource availability or higher quality patches in general. However, the conceptualization of the habitat-species relationship does not imply such a coincidence as a rule, although it permits it as a part of variation due to variability of habitat quality and to diversity of species traits. Instead, the conceptualization emphasizes the notion that specialization is a function of what is permitted in a given broader habitat unit. A permissible 
habitat may be good or poor and is, on balance, probably neutral when a large collection of species is considered.

A plausible consequence of the reduction of available resources along the gradient of specialization is an assumption that body size is negatively related to ecological range and, consequently, to the amount of habitat and its fragmentation (see Ziv, 2000 for details). While the generality of this assumption is yet to be proven, a number of studies show that habitat specialists tend to have smaller bodies (e.g., Kotze et al., 2003; Garcia-Barros and Benito, 2010; Symonds and Johnson, 2006 but see Hui and McGeoch, 2006 for a more complex picture that resembles one reported by Marquet et al., 1995). Additional theoretical and empirical evidence for this assumption has recently been provided by Tokeshi and Arakaki (2012) who showed that body size of aquatic invertebrates declines with fractal dimension of habitat texture - a strong correlate of habitat subdivision into small usable fragments.

\section{Formal model of habitat-species relationship}

In the most general sense we earlier proposed (Kolasa, 1989; Kolasa and Waltho, 1998) that

Species density $\propto k *$ (specialization $*$ habitat attributes)

where $k$ is an adjustment constant applied to the all species in a community under consideration; it adjusts the species density for the taxonomic group, habitat type, or any other intercommunity differences not covered by the remaining terms.

In community ecology species density is often and conveniently expressed as a share of total community abundance - relative density or abundance. The approach we advance here aims to obtain the relative density of a species with respect to the top generalist (cf., Fig. 2d). To do so we rely on a species' specialization as being an indicator of species performance (when made relative to the broadest range of ecological variables exploited by the least specialized member of that community; Figs. $2 \mathrm{~d}$ and 3 ). The maximum ecological range practically available to any species within a given species pool corresponds to the range of the top generalist in the habitat hierarchy, $S_{g}$. The ecological range of other species in that community will be denoted as $S_{i}$, The ratio of these two quantities $S_{i} / S_{g}$ gives the relative amount of habitat (resources) types available to species $i$. Species density, $N_{i}$, will also be affected systematically by the actual amount of the habitat, $H_{i}$, and by its fragmentation. The relative amount of the habitat available to species $i$ is evaluated by the ratio of $H_{i} / H_{g} . H_{i}$ or the habitat portion available to the species $i$ may be further broken into separate patches and thus exacerbate population penalties due to reduced habitat availability alone (cf., Fig. 2c). The latter can be expressed as $p_{i}$, where $p_{i}$ is the mean size of patches into which habitat of species $i\left(H_{i}\right)$ is meaningfully fragmented expressed as a fraction of the total habitat $H_{g}$ under consideration. Many different measures of fragmentation exist but all have major limitations (Fahrig, 2003). Mean number of patches in combination with the total amount of habitat available to species $i$ offers a sensible approximation of both the breakup and limited access or loss of habitat (fragmentation effect). Indeed, there are other aspects of fragmentation that may modify the predictions. Mean isolation and connectivity arising from patch shape, position, or the nature of habitat matrix may all increase or decrease the fragmentation effects (e.g., Fahrig, 2003) but such refinements are not the focus of the general model. Finally, the model can be made more realistic by adjusting the predicted abundance for allometric regularities such as a negative relationship between body size and population density. If necessary, other factors affecting the expected density such as species trophic position or metacommunity dynamics (unusually limited or strong dispersal ability) can also be factored in if necessary.
Thus, when the habitat specialization of species $i$ and the fragmentation of its habitat are known, we propose to calculate its abundance as:

$N_{i}=\frac{k}{M^{b}}\left(\frac{S_{i}}{S_{g}} * \frac{H_{i}}{H_{g}} * p_{i}\right)$

where $N_{i}$ (scaled between 0 and 1 ) is the relative density of species $i$ of adult mass $M(\mathrm{~kg})$, with specialization of $S_{i}$, and $b$ is the allometric scaling coefficient empirically determined to approach 0.75 (e.g., Carbone and Gittleman, 2002) for samples including a large number of species. $S_{g}$ is the ecological range of the most general species in a community, $H_{i}$ is the portion of the habitat used by species $i$ and $p_{i}$ is the mean size of patches into which the habitat of species $i$ is effectively broken within an a priori defined unit of comparison. Depending on the kinds of organisms, severity of fragmentation, and dispersal abilities $p$ could be replaced by other measures such as $H_{i}$ over median patch size, for instance, to better approximate the effect of patchiness on the costs to species $i$. Finally, $k$ (in $\mathrm{kg}$ ) is an adjustment factor for any relevant modifier of the expected density, such as resource quality, trophic level, taxonomic group, or dispersal, that is not implicitly or explicitly included in terms describing the amount and fragmentation of the habitat.

To explore some consequences of this formalization, we assume that habitat specialists tend to have either smaller body size or lower population density or a combination of both to reflect and compensate for an indirect effect of declining habitat availability. For simplification, let us represent expected $N_{i}$ as linked to body mass via a power function and as being proportional to the relative amount of habitat, $H_{i}$, and fragmentation, $p_{i}$ :

$N_{i}=\frac{1}{M^{b}} * \frac{H_{i} * p_{i}}{H_{g}}$

The probability of local extinction of a stochastically varying population, $P_{e}$, is a function of $N$ such that:

$P_{e}=\left(\frac{d}{b}\right)^{N}$

where $d$ is the death rate and $b$ is the birth rate. The arithmetic relationship between the difference between $b$ and $d$ (or per capita increase, $r$ ) and the ratio $d$ over $b$ can be expressed as:

$\frac{d}{b}=(f+g * r)^{-1}$

where $f$ and $g$ are constants defined by mean values of death and birth rates. By substituting for $N$ (from Eq. (3.2)) and ignoring the intercept constant $f$ we can write:

$P_{e}=(g * r)^{\left(1 / M^{b} *\left(H_{i} * p_{\hat{i}}\right) /\left(H_{g}\right)\right)}$

Finally, by solving for $M$ we find that for the probability of extinction to remain constant for any species in a community, mass should decline with the decline of available habitat according to the coefficient:

$M_{i}=\sqrt[b]{H_{i} * p_{i} / \log _{g r}\left(P_{e}\right)} * H_{g}$

The habitat-based model, HBM, outlined above (Eq. (3.1)), has a number of consequences. Some are novel predictions that are uniquely derived from this model while others address previously unsatisfactorily explained patterns (retrodictions). Below we review the model implications in two sections, community predictions and body size predictions.

\subsection{Community predictions}

\subsubsection{Accelerated decline of $N$ with the decrease of habitat range}

The habitat size (or multidimensional habitat volume) that is available to a species declines with species' specialization (cf., 
Fig. 3). From Eq. (3.1), species specialization is a relative term evaluated by the ratio $S_{i} / S_{g}$. When $S_{i}$ becomes narrower, the portion of habitat $H_{i} / H_{g}$ used by species $i$ also becomes smaller. Their product will lead to an accelerated decline of $N_{i}$. A commonly reported species range-abundance relationship (McGill, 2003; Julliard et al., 2006) is an inevitable consequence of this effect, regardless of whether or not other mechanisms contribute to its emergence (e.g., Holyoak and Loreau, 2006). Furthermore, the model explains why the slopes of range-abundance relationships are highly variable; they should be if the species specialization and habitat structure both differ from community to community. Mouquet and Loreau (2003) have approached the range-abundance question using a formal model, in which the main variables are dispersal and competitive abilities, and reached similar conclusions. This convergence may be due to the fact that the HBM focuses on heterogeneity (cf., Mouquet et al., 2006) and analyzes ultimate outcomes of dispersal, colonization success, competition, predation, and other, potentially indirect, constraints of species distribution and abundance.

\subsubsection{Increase in the relative variability of specialists}

Habitat specialists are commonly believed to be more vulnerable to environmental change, which may affect their population variability (Munday, 2004). HBM provides a theoretical justification for this idea. The primary argument in support of this prediction is couched in hierarchy theory. The theory posits (O'Neill et al., 1986) that lower level components operate at higher frequencies (with respect to the community, this implies rate of population or occupancy change) than the higher level components (generalists; Fig. 3). Thus, for a given window of observation, the relative variability of specialists should be higher than that of the generalists (details in Waltho and Kolasa, 1994). This prediction has received empirical support for fish (Waltho and Kolasa, 1994), aquatic invertebrates inhabiting rock pool microcosms (Kolasa et al., 1996; Kolasa and Li, 2003) and birds (Devictor et al., 2008). Possible mechanisms for the increased variability of specialists relative to habitat generalists may include the portfolio effect where the population of a generalist spans a number of different habitats in which its performance may be different and asynchronous (cf., Micheli et al., 1999) as opposed to a species restricted to a more homogeneous habitat space. Local variation of generalist competitors or predators may have a significant impact on a specialist utilizing the same local habitat space (e.g., Julliard et al., 2006), and lower density of specialists is subject to stochastic variation to a greater degree than that of the more abundant generalists (e.g., Munday, 2004).

All these mechanisms are consistent with the general prediction of the HBM and, indeed, hierarchy theory in general. Furthermore, it is quite possible, if not likely, that in most situations these mechanisms contribute jointly to the relative variability of species in any community. This differential variability along the gradient of specialization may have numerous positive and negative consequences that require separate examination.

\subsubsection{Increased probability of extinction of specialists}

Increased variability of specialists can be extended to infer their greater probability of extinction and to suggest several classes of mechanisms involved (cf., Eq. (3.5) in which the probability of extinction increases when habitat fraction available to a species, $H_{i}$, declines). The evidence ranges from patterns of survival in geological time (e.g., Jablonski, 1986), direct censuses of aquatic invertebrates (e.g., Benstead et al., 2003), flies (Worthen et al., 1998), butterflies (Charrette et al., 2006; Krauss et al., 2003), coral reef fish (Munday, 2004) and many others.

Possible mechanisms for the increased extinction rates of specialists relative to habitat generalists may include habitat restrictions in the face of large-scale environmental fluctuations (e.g., Charrette et al., 2006), stochastic consequences of small and isolated populations (e.g., in bighorn sheep; Festa-Bianchet et al., 2006), asymmetric interactions such as concentrated predation by a generalist predator, and shorter 'life span' of small habitat fragments (Boughton and Malvadkar, 2002).

\subsubsection{Predators (carnivores) should show a decrease of population energy use with body size as compared to omnivores}

Carbone and Gittleman (2002) identified this pattern empirically and provided a suite of energetic considerations such as local resource availability to account for the observed regularity. This includes Kleiber's law that a limited amount of available energy per unit area will sustain a larger number of individuals of a small-sized species than of a bigger species. However, it is possible to derive the qualitative aspects of this prediction from the HBM. The prediction combines known allometric regularities with relationships implied in the habitat-based model. The allometric expectation is that the density of both herbivores and carnivores declines with body size. The body size of prey and predators is generally correlated, with large predators tending (with some exceptions in aquatic systems) to consume larger prey (at least in energetically meaningful quantities; Romanuk et al., 2011). Thus, as the body size of predators increases, so does their prey. Habitat-based considerations imply that a decline of prey density $(\mathrm{Hi})$ will have a negative effect on its predator due to costs of resource fragmentation. The two trends can be combined.

From Eq. (3.1), $H_{i} / H_{g}$ reflects the costs of fragmentation due to declining amount of usable resource. Thus, if a prey is resource for the predator, $H_{i p}$ of the predator will be inversely related to the mass of its prey, $M_{h}$, because large prey are less abundant:

$$
H_{i p}=\frac{1}{M_{h}^{b h}}
$$

By substituting this and rearranging terms in Eq. (3.1) we obtain density of predator:

$N_{i p}=\frac{k}{M_{p}^{b p} * M_{h}^{b h} * H_{g}}\left(\frac{S_{i}}{S_{g}} * p_{i}\right)$

where $b p$ and $b h$ are the scaling coefficients for the predator and prey density with body mass, respectively.

A simple simulation illustrates the differential effect of increasing body size on prey and predator (Fig. 4). This prediction has empirical support from studies on mammals (Marquet et al., 1995; Carbone and Gittleman, 2002), in which predators showed more rapid decline in population density than omnivores as body size increased.

\subsubsection{Populations densities should show a triangular pattern of distribution as a function of body size}

Marquet et al. (1995) found for mammals and intertidal invertebrates that medium-size species attain higher population densities, and that population density decreases towards larger and smaller sizes. Here, we use Eq. (3.1) with an additional assumption that body size is negatively related to ecological range $\left(S_{i}\right)$ and, consequently, to $H_{i}$ as expressed in Eq. (3.6). Furthermore, we add random variation to $k$ to generate noise typical of larger data sets.

The general expectation is that reduction of habitat (as well as a possible increase in fragmentation) will dominate and reverse any benefits the smaller species may derive from energetic considerations alone and that energetic limitations will dominate large organisms. A similar expectation applies to larger-bodied species. In spite of their reduced metapopulation costs due to the increase in the available habitat and to the reduction of effective habitat fragmentation that is associated with the larger body size, the 


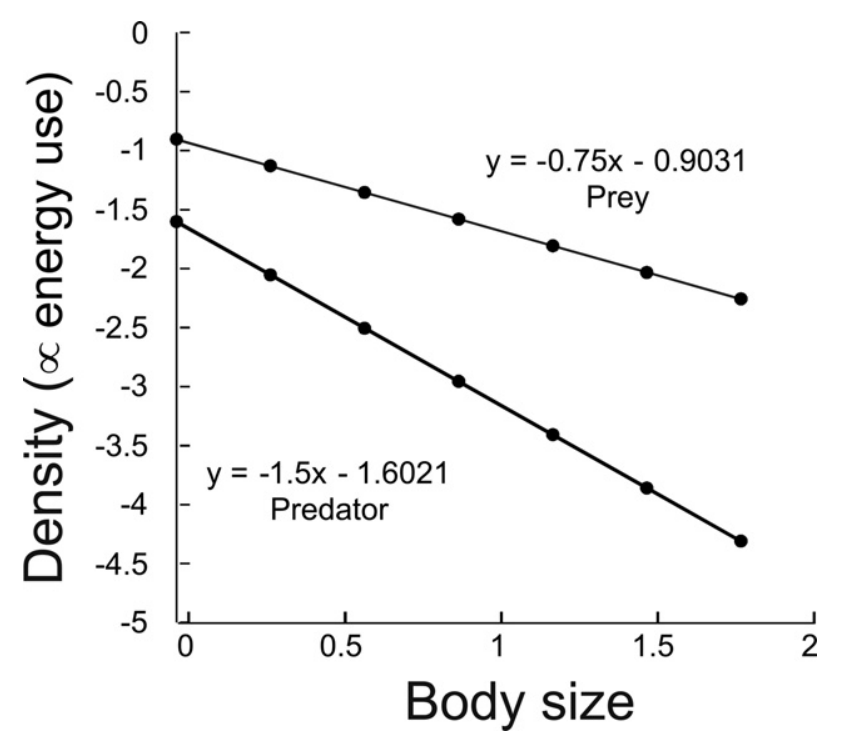

Fig. 4. Log-log relationship between body mass and density for prey (herbivore or omnivore) and its predator of the same size show a significant difference in slopes. Specialization, $k$, and patchiness were held constant. Furthermore, if one permits $S_{i}$ (ecological range) to increase with body mass, realistic slopes of about -0.9 for predator (cf., Carbone and Gittleman, 2002) are easily approached depending on the specific choice of parameters, for example, by making 'habitat landscape' use more generalized with increasing body size.

large-bodied species should experience a reduction in density because of the energetic constraints.

A simple simulation reproduces qualitative and unique aspects of the pattern observed in nature (left skewed distribution of species densities as a function of body size) (Fig. 5). In addition to addressing a specific pattern, this particular implementation of HBM offers an example of how change of scale leads to a shift in mechanisms. Here, depending on the resolution at which a species perceives its habitat, we see a shift in importance from spatial aspects of the habitat that primarily affects small species to the importance of energetic limitations that primarily affect mean density of large species.

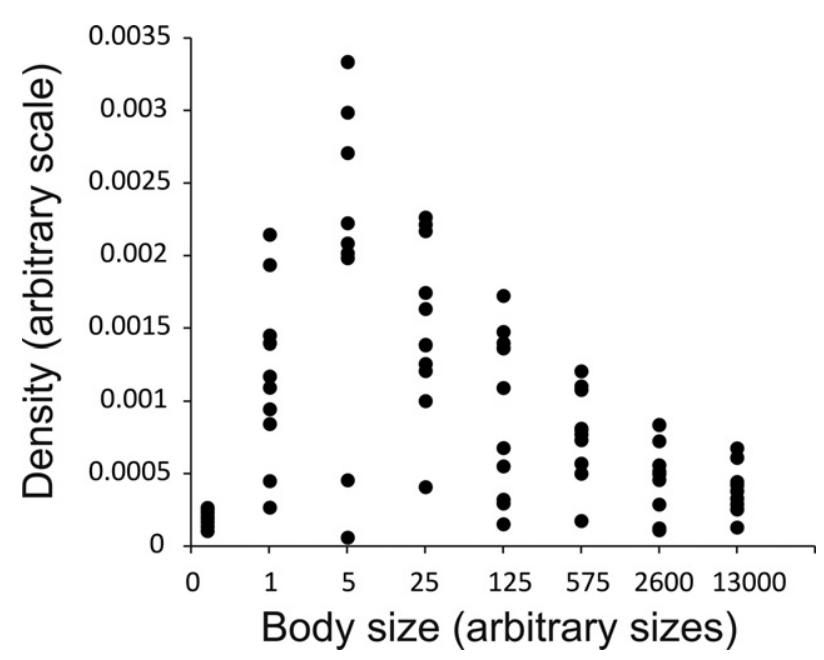

Fig. 5. A triangular distribution of species densities as a function of body size predicted by HBM: the graph is a composite of body size-density relationships for 80 species. These 80 species represent 10 communities with 8 species each and with $k$ varying randomly $(0,1)$ for each species in each community. Eq. (3.1) was used to calculate $\mathrm{Ni}$ and $\mathrm{M}$ was varied for all 10 communities by five orders of magnitude (from 0.2 to 13,000 of $\mathrm{kg}$ ). The same general pattern obtains irrespective of the value of the allometric scaling exponent $b(0.75$ or 0.67$)$ that relates body mass to density. Population density is relative.

\subsubsection{Fragmentation may benefit predator or prey depending on whose fragmentation cost is higher}

As habitat fragmentation increases (in Eq. (3.1), either $S_{i}$ (and, most likely, $H_{i}$ ) or $p_{i}$ decreases, or both changes take place), costs to populations increase. As shown earlier, predator $N_{i p}$ depends on habitat of its prey and declines faster than its prey, $N_{i h}$ (Fig. 4 ). However, what constitutes an effective fragmentation for prey may not represent fragmentation for a generalist predator that can use alternative prey (e.g., Swihart et al., 2001). In such a situation a predator - a generalist species in the sense of HBM model - may be affected indirectly by prey density decline only. Alternatively, equilibrial prey occupancy may increase even as habitat destruction increases; i.e., prey's "escape" from predation is facilitated by habitat loss when the risk of extinction due to predation is greater than a risk due to habitat fragmentation (Swihart et al., 2001, see also Eq. (3.5) for the effect of $H_{i}$ and probability of population extinction).

\subsubsection{Temporal specialization should be an effective escape from predation}

Restricting a species to a narrower use of habitat $\left(S_{i}\right)$ leads to a decrease in its abundance (in the form of a negative exponential function (Eq. (3.1)). Prey might be seen as an adaptive habitat that has the capacity to capitalize on this effect by restricting the predator's ecological $H_{i p}$ along the temporal axis. Thus, whenever prey occurrence can be pulsed as opposed to maintaining a constant level near the mean, $S_{i}$ of predator effectively decreases and results in a linear decrease in its abundance (Eq. (3.1)). One pulse of prey followed by an equal length period of no-prey would reduce $N_{p}$ to one-fourth and result in a corresponding decrease of predation on the prey population, $N_{h}$. Evidence for this mechanism in nature is ample, with the classical examples involving freshwater plankton prey and predators (e.g., Brandl, 2005), but notable exceptions have also been reported by Halle (1993) for European microtine rodents whose predators adapt their hunting in response to rodent activities. Spatial aggregation of individuals is a well-established predator defense mechanism (e.g., Coleman et al., 2004; but see Abrams, 2007 who does not introduce fragmentation and specializations costs in his model) that acts in a way similar to temporal specialization by changing effective $H_{\text {ip. }}$

\subsubsection{Perturbation to existing habitat structure will lead to an increase of habitat generalists relative to specialists}

This prediction can be deduced from modifications to Fig. 3. According to the convention employed to represent habitat structure, perturbation is introduced by either (i) a partial reduction of at least one of the two dimensions or (ii), creating additional subunits within those two dimensions. The reduction in one dimension means that the range of values along the axis representing that dimension becomes narrower and thus some values needed to produce specific habitat units will temporarily or permanently become unavailable. This is simply to say that perturbation will reduce the heterogeneity of the lower levels of the structure (cf., Kolasa and Rollo, 1991). Such reduction will in turn lead to the loss of specialist species using the smaller habitat units. Indeed, the total amount of habitat remains unchanged - it is only its diversification that is partially or entirely lost. Unless the disturbance produces an entirely new habitat type that is also unsuitable for generalists, generalists will increase in numbers at the expense of lost or suppressed specialists. Studies by Collins (1992) and Rooney et al., 2004 on plant communities provide convincing examples of this process. Alternatively, any existing subunit can be further subdivided. Such subdivision will reduce the relative abundance of the specialists at the level this subunit appears and thus will effectively increase the relative abundance of generalists. For example, the relative abundance 
of the generalist in Fig. 2d will increase from $67 \%$ to $73-76 \%$ if one of the subunits used by specialists is divided into unperturbed and perturbed portions (the perturbed portion can be occupied or not by a third specialist, which accounts for the range of the predicted values).

\subsection{Body size predictions}

\subsubsection{Discontinuities in body size distributions}

HBM representation of habitat-species relationship implies that species attributes such as body size, reproductive and dispersal strategies, and many others show abrupt transitions or even gaps (discontinuities) between ranges of similar values. Empirical support for this prediction is quite ample (reviewed in Stow et al., 2007). Notably, HBM also implies that empirical support should become increasingly confounded with the inclusion of larger geographic areas that may be home to habitats of differing structures. Some support for this implication is found in Siemann and Brown (1999).

We derive this prediction by combining assumptions about the habitat structure with assumptions of how organism size is related to decreasing usable habitat space. The idealized habitat structure shown in Fig. 3 assumes nesting of habitat units observed at one level of perceptual resolution within the next higher-level units. Nested condition is only met when the higher-level habitat unit consists of at least two, usually more, subunits. The inevitable consequence of the shift in habitat resolution is that the average habitat space available to species that use higher resolution is half or less than that available to a generalist using higher habitat unit (lower resolution). As seen in Figs. 1 and 3, hierarchy of habitat implies existence of clusters of habitat units that are in their own 'size' classes, corresponding to the level or 'scale' of the habitat, and with smaller habitats yielding larger clusters. Being specialized in using smaller habitat units has consequences: The amount of resources declines and thus the population density declines (see Eq. (3.1)). Both factors increase the probability of species extinction (Eq. (3.5)). To offset these negative consequences, two adaptive alternatives are available. One is to reduce the cost of finding and moving between the required habitat units. The other is to reduce the body mass to increase the size of effective population (cf., Eq. (3.6)). The latter is commonly observed as dwarfism in insular species that were once quite large (Lomolino, 2005). Assuming that body size decreases with habitat unit size used by a species (e.g., Ziv, 2000; Kotze et al., 2003; Garcia-Barros and Benito, 2010; Symonds and Johnson, 2006), discontinuous body size distributions are an inevitable consequence (Fig. 6).

\subsubsection{Greatest community changes correspond to discontinuities separating habitat units}

As presented above, recent research indicates that species using the same general landscape tend to occur in clusters of similar body sizes separated from other such clusters by distinct gaps (Sendzimir, 2008; Garmestani et al., 2009). But there is more to these size clusters than just their distinctness: species at the edges of clusters (those nearest discontinuities) appear to vary in various characteristics such as probability of extinction, population size, or some other metrics, more than species at the interior of lumps (Allen et al., 1999; Allen and Saunders, 2002; Wardwell and Allen, 2009; Allen and Holling, 2010).

This prediction, unlike those discussed earlier, requires additional consideration. All the previous predictions were direct consequences of the interaction of species with the habitat structure and modulated by the energetic consequences of body size. However the greater variability of species at the edges of body size clumps requires considering habitat variability as well. This variability has not been explicitly included in HBM but it can be.

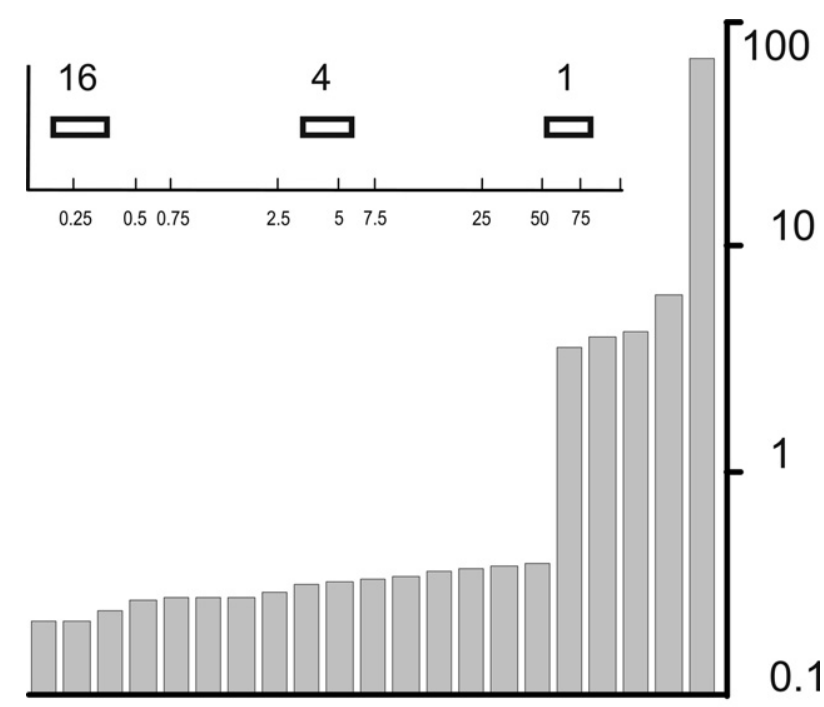

$\begin{array}{lllllllllll}1 & 3 & 5 & 7 & 9 & 11 & 13 & 15 & 17 & 19 & 21\end{array}$

Fig. 6. An example of body size distribution (log scale) in a hypothetical habitat with structure as shown in Fig. 3, calculated using Eq. (3.1), with added random variation $(0.5,1)$. The insert shows size ranges (rectangles) for each of the four species groups. The size range for the largest body size is a composite of 10 simulations while it is the average for the two smaller clumps. Numbers above rectangles give the number of species in each group.

We can derive the empirical observation made by Garmestani et al. (2009) from HBM in conjunction with the idea that organism size is related to the size of the habitat unit a species uses (cf., Eq. (3.6)). Within any cluster of species sizes some species will be the smaller and some larger. Let's now consider consequences of environmental variation. If the environment changes, the ecological space of any species will also change: previously inaccessible habitat space may become accessible or some habitat space may become inaccessible (as in anticipated range shifts due to climate warming). Each species in a defined size group can theoretically expand or shrink its habitat use. In terms of the graphical model (Figs. 2 and 3), each species can potentially add ecological space at the expense of a habitat unit that was previously inaccessible but became accessible as a result of the change in habitat quality. Such a species can thus expand its $H_{i}$. A species can also lose a portion of its habitat and be even demoted to the next lower level if the loss is substantial and the lost habitat is replaced with a different type. This is similar to a prediction that population density should vary most at the edge of geographical range of a species whenever that range is limited by environmental conditions (e.g., Curnutt et al., 1996; Williams et al., 2003). A simple simulation of random habitat change for habitat structure shown in Fig. 3 shows that the smallest habitat units at each level of resolution undergo the greatest relative variation ( $51 \%$ and $83 \%$ for the intermediate and lowest level patches, respectively; Fig. 7). Such a variation can be assumed to correlate with variation of the associated species, which, in this case would most likely be the smallest species in a cluster. Notably, the variation distribution reproduces empirical patterns reported elsewhere (e.g., Waltho and Kolasa, 1994; Kolasa and Li, 2003) where variability of specialists (coral reef fish and rock pool invertebrates) has been shown to exceed greatly that of habitat generalists.

\section{General observations}

Many of the patterns predicted by Habitat Based Model can be derived from a variety of other, unrelated, models. For example, the positive correlation between species distribution and density 


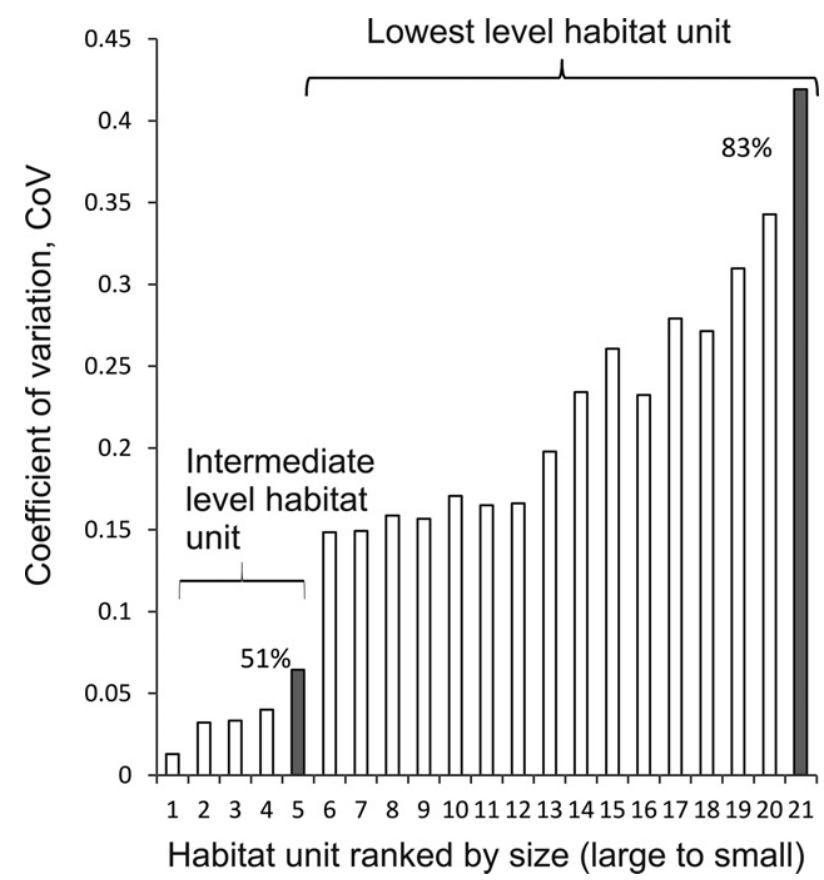

Fig. 7. Variation of habitat units is greatest at scale transitions (Units 5 and 21, shaded). Brackets show groups of habitats at one scale of resolution; Unit 1 is the largest habitat (top level in Fig. 3). Percentages are differences from the group mean coefficients of variation. Data based on 50 simulations of random change in size applied to every habitat unit at each level (the same arbitrary mean change for all the units).

is predicted by metacommunity models as well as by neutral theory (Hubbell, 2001). Statistical models considering sampling probability also link abundance and occurrence (He and Gaston, 2000). Energetic models and fractal distribution models lead to similar results. However, none of these alternative explanatory frameworks is capable of predicting discontinuities (but see Hanski, 1982 for a model predicting bimodal distribution). More importantly, none of these frameworks offers the scope of consequences and the universality of assumptions (habitat has structure and species differ from one another) to infer observable patterns. Thus, in relative terms HBM is the most parsimonious model and the most fundamental model that draws on the logic employed by Darwin (that species change and that change has to do with habitat limitations). Finally, unlike the great majority of the available models, HBM addresses explicitly shifts in scale and their consequences, an inherent attribute of ecological systems. HBM has affinities and converges in some respects with the work done within a metacommunity framework, particularly regarding the consequences of heterogeneity and dispersal (e.g., Mouquet et al., 2006; Mouquet and Loreau, 2003).

Perhaps the most novel feature of the HBM perspective is that it underscores the presence of discontinuities in characteristics of ecological systems and their processes. Such discontinuities may be found in the traits of organisms making up a community, in their ranked abundances, in characteristic frequencies at which processes operate (cf., hierarchy theory by O'Neill et al., 1986), or in space.

\section{Conclusions}

We have presented the Habitat-Based model that conceptualizes habitat as a hierarchy of nested habitat units. We have attempted to show that the model offers a simple starting structure to exploration of relationships between species pools and the habitat they use, that it is quite good at these explorations, and that

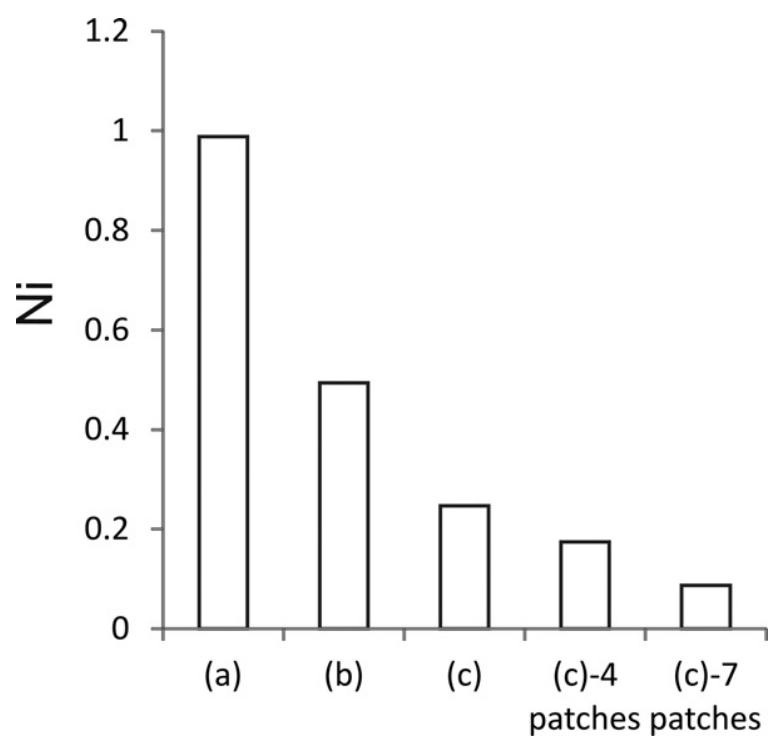

Fig. A1. Effect of fragmentation, $p_{i}$, on population size $N_{i}$, according to Eq. (3.1) where $p_{i}$ is a mean patch size. Calculations apply to Fig. $2 \mathrm{a}-\mathrm{c}$ and a situation where 8 small patches are reduced to 4 (case c less 4 patches) and then 1 (case c less 7 patches). When the habitat 'landscape' transforms from 8 to 4 (or 1) patches, mean patch isolation increases. The model does not include this effect directly as the effect of isolation is not universally negative. Furthermore, the amount of habitat, $H_{i}$, and the mean patch size, $p_{i}$, have a dominant effect on $N_{i}$. Specific effect of isolation could be factored in for individual species or habitat situations based on realistic estimates of the parameter but it does not seem necessary given the behavior of the model.

such explorations are not limited to just one set of problems. In fact, we would argue that the very scope and depth of the perspective make it the most parsimonious approach to many problems of community ecology and areas related to it. Rooting the model in the relationship between the habitat structure and species attributes allows it to evolve to address a range of concerns and research questions not discussed in this review.

\section{Acknowledgements}

McDonnell Foundation grant to CA, and NSERC grant to JK provided funding for this project. Jon Dushoff and Tamara Romanuk supplied helpful comments on the earlier version of the manuscript. The paper constitutes GLERL contribution number 1625; (C.A. Stow). The Nebraska Cooperative Fish and Wildlife Research Unit is jointly supported by a cooperative agreement between the U.S. Geological Survey, the Nebraska Game and Parks Commission, the University of Nebraska-Lincoln, the United States Fish and Wildlife Service, and the Wildlife Management Institute.

\section{Appendix A.}

See Fig. A1.

\section{References}

Abrams, P.A., 2007. Habitat choice in predator-prey systems: spatial instability due to interacting adaptive movements. American Naturalist 169, 581-594.

Allen, C.R., Forys, E.A., Holling, C.S., 1999. Body mass patterns predict invasions and extinctions in transforming landscapes. Ecosystems 2, 114-121.

Allen, C.R., Holling, C.S., 2010. Novelty, adaptive capacity, and resilience. Ecology and Society $15,24-34$.

Allen, C.R., Saunders, D.A., 2002. Variability between scales: predictors of nomadism in birds of an Australian Mediterranean-climate ecosystem. Ecosystems 5, 348-359.

Allen, T.F.H., Starr, T.B., 1982. Hierarchy: Perspectives for Ecological Complexity. The University of Chicago Press, Chicago. 
Benstead, J.P., Douglas, M.M., Pringle, C.M., 2003. Relationships of stream invertebrate communities to deforestation in eastern Madagascar. Ecological Applications 13, 1473-1490.

Boughton, D., Malvadkar, U., 2002. Extinction risk in successional landscapes subject to catastrophic disturbances. Conservation Ecology 6, 1-24.

Brandl, Z., 2005. Freshwater copepods and rotifers: predators and their prey. Hydrobiologia 546, 475-489.

Carbone, C., Gittleman, J.L., 2002. A common rule for the scaling of carnivore density. Science 295, 2273-2276.

Charrette, N.A., Cleary, D.F.R., Mooers, A.O., 2006. Range-restricted, specialist Bornean butterflies are less likely to recover from enso-induced disturbance. Ecology 87, 2330-2337.

Coleman, R.A., Browne, M., Theobalds, T., 2004. Aggregation as a defense: limpet tenacity changes in response to simulated predator attack. Ecology 85, 1153-1159.

Collins, S., 1992. Fire frequency and community heterogeneity in tallgrass prairie vegetation. Ecology 76, 2001-2006.

Curnutt, J.L., Pimm, S.L., Maurer, B.A., 1996. Population variability of sparrows in space and time. Oikos 76, 131-144.

Davies, K.F., Chesson, P., Harrison, S., Inouye, B.D., Melbourne, B.A., Rice, K.J., 2005. Spatial heterogeneity explains the scale dependence of the native-exotic diversity relationship. Ecology 86, 1602-1610.

Devictor, V., Julliard, R., Frédéric, J., 2008. Distribution of specialist and generalist species along spatial gradients of habitat disturbance and fragmentation. Oikos $117,507-514$.

Fahrig, L., 2003. Effects of habitat fragmentation on biodiversity. Annual Review of Ecology, Evolution and Systematics 34, 487-515.

Festa-Bianchet, M., Coulson, T., Gaillard, J.M., Hogg, J.T., Pelletier, F., 2006. Stochastic predation events and population persistence in bighorn sheep. Proceedings of the Royal Society B: Biological Sciences 273, 1537-1543.

Garcia-Barros, E., Benito, H.R., 2010. The relationship between geographic range size and life history traits: is biogeographic history uncovered? A test using the Iberian butterflies. Ecography 33, 392-401.

Garmestani, A.S., Allen, C.R., Gunderson, L., 2009. Panarchy discontinuities reveal similarities in the dynamic system structure of ecological and social systems. Ecology and Society, 14, online http://www.ecologyandsociety. org/vol14/iss1/art15/.

Halle, S., 1993. Diel pattern of predation risk in microtine rodents. Oikos 68, 510-518.

Hanski, I., 1982. Dynamics of regional distribution: the core and satellite species hypothesis. Oikos 38, 210-221.

He, F., Gaston, K.J., 2000. Occupancy-abundance relationships and sampling scales. Ecography 23, 503-511.

Holling, C.S., 1992. Cross-scale morphology, geometry, and dynamics of ecosystems. Ecological Monographs 62, 447-502.

Holyoak, M., Loreau, M., 2006. Reconciling empirical ecology with neutral community models. Ecology 87, 1370-1377.

Hubbell, S.P., 2001. The Unified Neutral Theory of Biodiversity and Biogeography. Princeton University Press, Princeton.

Hui, C., McGeoch, M.A., 2006. Evolution of body size, range size, and food composition in a predator-prey metapopulation. Ecological Complex 3,148-159.

Jablonski, D., 1986. Background and mass extinctions: the alternation of macroevolutionary regimes. Science 231, 129-133.

Julliard, R., Clavel, J., Devictor, V., Jiguet, F., Couvet, D., 2006. Spatial segregation of specialists and generalists in bird communities. Ecology Letters 9, 1237-1244.

Kolasa, J., 1989. Ecological systems in hierarchical perspective: breaks in the community structure and other consequences. Ecology 70, 36-47.

Kolasa, J., Drake, J.A., Huxel, G.R., Hewitt, C.L., 1996. Hierarchy underlies patterns of variability in species inhabiting natural microcosms. Oikos 77, 259-266.

Kolasa, J., Li, B-L., 2003. Removing the confounding effect of habitat specialization reveals stabilizing contribution of diversity to species variability. Biology Letters 9, 1-4.

Kolasa, J., Rollo, D.C., 1991. Heterogeneity of heterogeneity: a glossary. In: Kolasa, J., Pickett, S.T.A. (Eds.), Ecological Heterogeneity. Springer-Verlag, New York, pp. $1-23$.
Kolasa, J., Waltho, N., 1998. A hierarchical view of habitat and its relation to species abundance. In: Peterson, D., Parker, V.T. (Eds.), Ecological Scale: Theory and Applications. Columbia University Press, New York, pp. 53-76.

Kotze, D.J., Niemela, J., O’Hara, R.B., Turin, H., 2003. Testing abundance-range size relationships in European carabid beetles (Coleoptera, Carabidae). Ecography $26,553-566$.

Krauss, J., Steffan-Dewenter, I., Tscharntke, T., 2003. Local species immigration, extinction, and turnover of butterflies in relation to habitat area and habitat isolation. Oecologia 137, 591-602.

Lomolino, M.V., 2005. Body size evolution in insular vertebrates: generality of the island rule. Journal of Biogeography 32, 1683-1699.

Marquet, P.A., Navarrete, S.A., Castilla, J.C., 1995. Body-size, population-density, and the energetic equivalence rule. Journal of Animal Ecology 64, 325-332.

McGill, B., 2003. A test of the unified neutral theory of biodiversity. Nature 422 , 881-885.

Micheli, F., Cottingham, K.L., Bascompte, J., Bjørnstad, O.N., Eckert, G.L., Fischer, J.M., et al., 1999. The dual nature of community variability. Oikos 85, 161-169.

Mouquet, N., Loreau, M., 2003. Community patterns in source-sink metacommunities. American Naturalist 162, 544-557.

Mouquet, N., Miller, T.E., Daufresne, T., Kneitel, J.M., 2006. Consequences of varying regional heterogeneity in source-sink metacommunities. Oikos 113, 481-488.

Munday, P.L., 2004. Habitat loss, resource specialization, and extinction on coral reefs. Global Change Biology 10, 1642-1647.

O'Neill, R.V., DeAngelis, D.L., Waide, J.B., Allen, T.F.H., 1986. A Hierarchical Concept of Ecosystems. Princeton University Press, Princeton.

Polechová, J., Storch, D., 2008. Ecological niche. In: Jørgensen, S.K., Fath, B.D. (Eds.), Encyclopedia of Ecology, vol. 2. Elsevier, Oxford, pp. 1088-1097.

Romanuk, T.N., Hayward, A., Hutchings, J.A., 2011. Trophic level scales positively with body size in fishes. Global Ecology and Biogeography 20, 231-240.

Rooney, T.P., Wiegmann, S.M., Rogers, D.A., Waller, D.M., 2004. Biotic impoverishment and homogenization in unfragmented forest understory communities Conservation Biology 18, 787-798.

Sandin, S.A., Pacala, S.W., 2005. Fish aggregation results in inversely densitydependent predation on continuous coral reefs. Ecology 86, 1520-1530.

Sendzimir, J., 2008. Patterns of landscape structure, discontinuity, mammal phylogeny, and body size. In: Allen, C.R., Holling, C.S. (Eds.), Discontinuities in Ecosystems and Other Complex Systems. Columbia University Press, New York.

Siemann, E., Brown, J.H., 1999. Gaps in mammalian body size distributions reexamined. Ecology 80, 2788-2792.

Stow, C., Allen, C.R., Garmestani, A.S., 2007. Evaluating discontinuities in complex systems: toward quantitative measures of resilience. Ecology and Society, 12 (1), online http://www.ecologyandsociety.org/vol12/iss1/art26/.

Swihart, R.K., Feng, Z.L., Slade, N.A., Mason, D.M., Gehring, T.M., 2001. Effects of habitat destruction and resource supplementation in a predator-prey metapopulation model. Journal of Theoretical Biology 210, 287-303.

Symonds, M.R.E., Johnson, C.N., 2006. Determinants of local abundance in a major radiation of Australian passerines (Aves: Meliphagoidea). Journal of Biogeography 33, 794-802.

Tokeshi, M., Arakaki, S., 2012. Habitat complexity in aquatic systems: fractals and beyond. Hydrobiologia 685, 27-47.

Waltho, N., Kolasa, J., 1994. Organization of instabilities in multispecies systems: a test of hierarchy theory. Proceedings of the National Academy of Sciences of the United States of America 91, 1682-1685.

Wardwell, D., Allen, C.R., 2009. Variability in population abundance is associated with thresholds between scaling regimes. Ecology and Society, 14, online http://www.ecologyandsociety.org/vol14/iss2/art42/.

Williams, C.K., Ives, A.R., Applegate, R.D., 2003. Population dynamics across geographical ranges: time-series analyses of three small game species. Ecology 84, 2654-2667.

Worthen, W.B., Jones, T.M., Jetton, R.M., 1998. Community structure and environmental stress: desiccation promotes nestedness in mycophagous fly communities. Oikos 81, 45-54.

Ziv, Y., 2000. On the scaling of habitat specificity with body size. Ecology 81 2932-2938. 